\title{
Complex Convexity of Musielak-Orlicz Function Spaces Equipped with the $p$-Amemiya Norm
}

\author{
Lili Chen, Yunan Cui, and Yanfeng Zhao \\ Department of Mathematics, Harbin University of Science and Technology, Harbin 150080, China \\ Correspondence should be addressed to Lili Chen; cll2119@hotmail.com
}

Received 27 November 2013; Revised 5 April 2014; Accepted 13 April 2014; Published 8 May 2014

Academic Editor: Angelo Favini

Copyright (C) 2014 Lili Chen et al. This is an open access article distributed under the Creative Commons Attribution License, which permits unrestricted use, distribution, and reproduction in any medium, provided the original work is properly cited.

The complex convexity of Musielak-Orlicz function spaces equipped with the $p$-Amemiya norm is mainly discussed. It is obtained that, for any Musielak-Orlicz function space equipped with the $p$-Amemiya norm when $1 \leq p<\infty$, complex strongly extreme points of the unit ball coincide with complex extreme points of the unit ball. Moreover, criteria for them in above spaces are given. Criteria for complex strict convexity and complex midpoint locally uniform convexity of above spaces are also deduced.

\section{Introduction}

Let $(X,\|\cdot\|)$ be a complex Banach space over the complex field $\mathbb{C}$, let $i$ be the complex number satisfying $i^{2}=-1$, and let $B(X)$ and $S(X)$ be the closed unit ball and the unit sphere of $X$, respectively. In the sequel, $\mathbb{N}$ and $\mathbb{R}$ denote the set of natural numbers and the set of real numbers, respectively.

In the early 1980s, a huge number of papers in the area of the geometry of Banach spaces were directed to the complex geometry of complex Banach spaces. It is well known that the complex geometric properties of complex Banach spaces have applications in various branches, among others in Harmonic Analysis Theory, Operator Theory, Banach Algebras, $C^{*}$ Algebras, Differential Equation Theory, Quantum Mechanics Theory, and Hydrodynamics Theory. It is also known that extreme points which are connected with strict convexity of the whole spaces are the most basic and important geometric points in geometric theory of Banach spaces (see [1-6]).

In [7], Thorp and Whitley first introduced the concepts of complex extreme point and complex strict convexity when they studied the conditions under which the Strong Maximum Modulus Theorem for analytic functions always holds in a complex Banach space.

A point $x \in S(X)$ is said to be a complex extreme point of $B(X)$ if for every nonzero $y \in X$ there holds $\sup _{|\lambda| \leq 1}\|x+\lambda y\|>$ 1. A complex Banach space $X$ is said to be complex strictly convex if every element of $S(X)$ is a complex extreme point of $B(X)$.
In [8], we further studied the notions of complex strongly extreme point and complex midpoint locally uniform convexity in general complex spaces.

A point $x \in S(X)$ is said to be a complex strongly extreme point of $B(X)$ if for every $\varepsilon>0$ we have $\Delta_{c}(x, \varepsilon)>0$, where

$$
\begin{gathered}
\Delta_{c}(x, \varepsilon)=\inf \{1-|\lambda|: \exists y \in X \text { s.t. }\|\lambda x \pm y\| \leq 1, \\
\|\lambda x \pm i y\| \leq 1,\|y\| \geq \varepsilon\} .
\end{gathered}
$$

A complex Banach space $X$ is said to be complex midpoint locally uniformly convex if every element of $S(X)$ is a complex strongly extreme point of $B(X)$.

Let $(T, \Sigma, \mu)$ be a nonatomic and complete measure space with $\mu(T)<\infty$. By $\Phi$ we denote a Musielak-Orlicz function; that is, $\Phi: T \times[0,+\infty) \rightarrow[0,+\infty]$ satisfies the following:

(1) for each $u \in[0, \infty], \Phi(t, u)$ is a $\mu$-measurable function of $t$ on $T$;

(2) for $\mu$-a.e. $t \in T, \Phi(t, 0)=0, \lim _{u \rightarrow \infty} \Phi(t, u)=\infty$ and there exists $u^{\prime}>0$ such that $\Phi\left(t, u^{\prime}\right)<\infty$;

(3) for $\mu$-a.e. $t \in T, \Phi(t, u)$ is convex on the interval $[0, \infty)$ with respect to $u$.

Let $L^{c}(\mu)$ be the space of all $\mu$-equivalence classes of complex and $\Sigma$-measurable functions defined on $T$. For each $x \in L^{c}(\mu)$, we define on $L^{c}(\mu)$ the convex modular of $x$ by

$$
I_{\Phi}(x)=\int_{T} \Phi(t,|x(t)|) d t .
$$


We define supp $x=\{t \in T:|x(t)| \neq 0\}$ and the MusielakOrlicz space $L_{\Phi}$ generated by the formula

$$
L_{\Phi}=\left\{x \in L^{c}(\mu): I_{\Phi}(\lambda x)<\infty \text { for some } \lambda>0\right\} .
$$

Set

$$
\begin{aligned}
& e(t)=\sup \{u \geq 0: \Phi(t, u)=0\}, \\
& E(t)=\sup \{u \geq 0: \Phi(t, u)<\infty\} ;
\end{aligned}
$$

then $e(t)$ and $E(t)$ are $\mu$-measurable (see [9]).

The notion of $p$-Amemiya norm has been introduced in [10]; for any $1 \leq p \leq \infty$ and $u>0$, define

$$
s_{p}(u)= \begin{cases}\left(1+u^{p}\right)^{1 / p}, & \text { for } 1 \leq p<\infty \\ \max \{1, u\}, & \text { for } p=\infty\end{cases}
$$

Furthermore, define $s_{\Phi, p}(x)=s_{p} \circ I_{\Phi}(x)$ for all $1 \leq p \leq \infty$ and $x \in L^{c}(\mu)$. Notice that the function $s_{p}$ is increasing on $R_{+}$ for $1 \leq p<\infty$; however, the function $s_{\infty}$ is increasing on the interval $[1, \infty)$ only.

For $x \in L^{c}(\mu)$, define the $p$-Amemiya norm by the formula

$$
\|x\|_{\Phi, p}=\inf _{k>0} \frac{1}{k} s_{\Phi, p}(k x), \quad 1 \leq p \leq \infty
$$

For convenience, from now on, we write $L_{\Phi, p}=$ $\left(L_{\Phi},\|\cdot\|_{\Phi, p}\right)$; it is easy to see that $L_{\Phi, p}$ is a Banach space. For $1 \leq p<\infty, x \in L_{\Phi, p}$, set

$$
K_{p}(x)=\left\{k>0:\|x\|_{\Phi, p}=\frac{1}{k}\left\{1+I_{\Phi}^{p}(k x)\right\}^{1 / p}\right\} .
$$

\section{Main Results}

We begin this section from the following useful lemmas.

Lemma 1 (see [9]). For any $\varepsilon>0$, there exists $\delta \in(0,1 / 2)$ such that if $u, v \in \mathbb{C}$ and

$$
|v| \geq \frac{\varepsilon}{8} \max _{e}|u+e v|,
$$

then

$$
|u| \leq \frac{1-2 \delta}{4} \Sigma_{e}|u+e v|
$$

where

$$
\begin{gathered}
\max _{e}|u+e v|=\max \{|u+v|,|u-v|,|u+i v|,|u-i v|\}, \\
\Sigma_{e}|u+e v|=|u+v|+|u-v|+|u+i v|+|u-i v| .
\end{gathered}
$$

Lemma 2. If $\lim _{u \rightarrow \infty}(\Phi(t, u) / u)=\infty$ for $\mu$-a.e. $t \in T$, then $K_{p}(x) \neq \emptyset$ for any $x \in L_{\Phi, p} \backslash\{0\}$, where $1 \leq p<\infty$.

Proof. For any $x \in L_{\Phi, p} \backslash\{0\}$, there exists $\alpha>0$ such that $\mu\{t \in T: \quad|x(t)| \geq \alpha\}>0$. Let $T_{1}=\{t \in T: \quad|x(t)| \geq \alpha\}$ and define the subsets

$$
F_{n}=\left\{t \in T_{1}: \frac{\Phi(t, u)}{u} \geq \frac{4\|x\|_{\Phi, p}}{\alpha \cdot \mu T_{1}}, u \geq n\right\}
$$

for each $n \in \mathbb{N}$. It is easy to see that $F_{1} \subseteq F_{2} \subseteq \cdots \subseteq F_{n} \subseteq \cdots$ and $\lim _{n \rightarrow \infty} \mu F_{n}=\mu T_{1}$. Then there exists $n_{0} \in \mathbb{N}$ such that $\mu F_{n_{0}}>(1 / 2) \mu T_{1}$.

It follows from the definition of $p$-Amemiya norm that there is a sequence $\left\{k_{n}\right\}$ satisfying

$$
\|x\|_{\Phi, p}=\lim _{n \rightarrow \infty} \frac{1}{k_{n}}\left\{1+I_{\Phi}^{p}\left(k_{n} x\right)\right\}^{1 / p} .
$$

For any $k>0$, we have

$$
\begin{aligned}
& \frac{1}{k}\{1\left.+I_{\Phi}^{p}(k x)\right\}^{1 / p} \\
&=\frac{1}{k}\left\{1+\left[\int_{T} \Phi(t, k|x(t)|) d t\right]^{p}\right\}^{1 / p} \\
& \geq \frac{\int_{F_{n_{0}}} \Phi(t, k|x(t)|) d t}{k} \\
& \geq \int_{F_{n_{0}}} \frac{\Phi(t, k \alpha)}{k} d t \\
& \quad=\alpha \int_{F_{n_{0}}} \frac{\Phi(t, k \alpha)}{k \alpha} d t .
\end{aligned}
$$

If $k>n_{0} / \alpha$, notice that

$$
\frac{1}{k}\left\{1+I_{\Phi}^{p}(k x)\right\}^{1 / p} \geq \alpha \cdot \frac{4\|x\|_{\Phi, p}}{\alpha \cdot \mu T_{1}} \cdot \mu F_{n_{0}}>2\|x\|_{\Phi, p},
$$

which means the sequence $\left\{k_{n}\right\}$ is bounded. Hence, without loss of generality, we assume that $k_{n} \rightarrow k_{0}$ as $n \rightarrow \infty$. We can also choose the monotonic increasing or decreasing subsequence of $\left\{k_{n}\right\}$ that converges to the number $k_{0}$. Applying Levi Theorem and Lebesgue Dominated Convergence Theorem, we obtain

$$
\|x\|_{\Phi, p}=\lim _{n \rightarrow \infty} \frac{1}{k_{n}}\left\{1+I_{\Phi}^{p}\left(k_{n} x\right)\right\}^{1 / p}=\frac{1}{k_{0}}\left\{1+I_{\Phi}^{p}\left(k_{0} x\right)\right\}^{1 / p}
$$

which implies $k_{0} \in K_{p}(x)$.

In order to exclude the case when such sets $K_{p}(x)$ are empty for $x \in L_{\Phi, p} \backslash\{0\}$, in the sequel we will assume, without any special mention, that $\lim _{u \rightarrow \infty}(\Phi(t, u) / u)=\infty$ for $\mu$-a.e. $t \in T$.

Theorem 3. Assume $1 \leq p<\infty, x \in S\left(L_{\Phi, p}\right)$. Then the following assertions are equivalent:

(1) $x$ is a complex strongly extreme point of the unit ball $B\left(L_{\Phi, p}\right)$;

(2) $x$ is a complex extreme point of the unit ball $B\left(L_{\Phi, p}\right)$;

(3) for any $k \in K_{p}(x), \mu\{t \in T: k|x(t)|<e(t)\}=0$.

Proof. The implication (1) $\Rightarrow(2)$ is trivial. Let $x$ be a complex extreme point of the unit ball $B\left(L_{\Phi, p}\right)$ and there exists $k_{0} \in$ $K_{p}(x)$ such that $\mu\left\{t \in T: k_{0}|x(t)|<e(t)\right\}>0$. Then we can 
find $d>0$ such that $\mu\left\{t \in T: k_{0}|x(t)|+d<e(t)\right\}>0$. Indeed, if $\mu\left\{t \in T: k_{0}|x(t)|+d<e(t)\right\}=0$ for any $d>0$, then we set $d_{n}=1 / n$ and define the subsets

$$
T_{n}=\left\{t \in T: k_{0}|x(t)|+d_{n}<e(t)\right\}
$$

for each $n \in \mathbb{N}$. Notice that $T_{1} \subseteq T_{2} \subseteq \cdots \subseteq T_{n} \subseteq \cdots$ and $\left\{t \in T: \quad k_{0}|x(t)| \leq e(t)\right\}=\bigcup_{n=1}^{\infty} T_{n}$; we can find that

$$
\mu\left\{t \in T: k_{0}|x(t)| \leq e(t)\right\}=0
$$

which is a contradiction.

Let $T_{0}=\left\{t \in T: k_{0}|x(t)|+d<e(t)\right\}$ and define $y=$ $\left(d / k_{0}\right) \chi_{T_{0}}$; we have $y \neq 0$ and for any $\lambda \in \mathbb{C}$ with $|\lambda| \leq 1$,

$$
\begin{aligned}
\|x+\lambda y\|_{\Phi, p} \leq & \frac{1}{k_{0}}\left\{1+I_{\Phi}^{p}\left(k_{0}(x+\lambda y)\right)\right\}^{1 / p} \\
= & \frac{1}{k_{0}}\left\{1+\left[I_{\Phi}\left(k_{0} x \chi_{T \backslash T_{0}}\right)\right.\right. \\
& \left.\left.+I_{\Phi}\left(k_{0} x \chi_{T_{0}}+\lambda d \chi_{T_{0}}\right)\right]^{p}\right\}^{1 / p} \\
& \leq \frac{1}{k_{0}}\left\{1+\left[I_{\Phi}\left(k_{0} x \chi_{T \backslash T_{0}}\right)\right.\right. \\
& \left.\left.+I_{\Phi}\left(\left(k_{0}|x|+d\right) \chi_{T_{0}}\right)\right]^{p}\right\}^{1 / p} \\
= & \frac{1}{k_{0}}\left\{1+\left[I_{\Phi}\left(k_{0} x \chi_{T \backslash T_{0}}\right)\right]^{p}\right\}^{1 / p} \\
= & \frac{1}{k_{0}}\left(1+I_{\Phi}^{p}\left(k_{0} x\right)\right)^{1 / p}=1,
\end{aligned}
$$

which shows that $x$ is not a complex extreme point of the unit ball $B\left(L_{\Phi, p}\right)$.

(3) $\Rightarrow$ (1). Suppose that $x \in S\left(L_{\Phi, p}\right)$ is not a complex strongly extreme point of the unit ball $B\left(L_{\Phi, p}\right)$; then there exists $\varepsilon_{0}>0$ such that $\Delta_{c}\left(x, \varepsilon_{0}\right)=0$. That is, there exist $\lambda_{n} \in \mathbb{C}$ with $\left|\lambda_{n}\right| \rightarrow 1$ and $y_{n} \in L_{\Phi, p}$ satisfying $\left\|y_{n}\right\|_{\Phi, p} \geq \varepsilon_{0}$ such that

$$
\left\|\lambda_{n} x_{0} \pm y_{n}\right\|_{\Phi, p} \leq 1, \quad\left\|\lambda_{n} x_{0} \pm i y_{n}\right\|_{\Phi, p} \leq 1,
$$

which gives

$$
\left\|x_{0} \pm \frac{y_{n}}{\lambda_{n}}\right\|_{\Phi, p} \leq \frac{1}{\left|\lambda_{n}\right|}, \quad\left\|x_{0} \pm i \frac{y_{n}}{\lambda_{n}}\right\|_{\Phi, p} \leq \frac{1}{\left|\lambda_{n}\right|} .
$$

Setting $z_{n}=y_{n} / \lambda_{n}$, we have

$$
\begin{gathered}
\left\|z_{n}\right\|_{\Phi, p} \geq\left\|y_{n}\right\|_{\Phi, p} \geq \varepsilon_{0}, \\
\left\|x_{0} \pm z_{n}\right\|_{\Phi, p} \leq \frac{1}{\left|\lambda_{n}\right|}, \\
\left\|x_{0} \pm i z_{n}\right\|_{\Phi, p} \leq \frac{1}{\left|\lambda_{n}\right|} .
\end{gathered}
$$

For the above $\varepsilon_{0}>0$, by Lemma 1 , there exists $\delta_{0} \epsilon$ $(0,1 / 2)$ such that if $u, v \in \mathbb{C}$ and

$$
|v| \geq \frac{\varepsilon_{0}}{8} \max _{e}|u+e v|
$$

then

$$
|u| \leq \frac{1-2 \delta_{0}}{4} \Sigma_{e}|u+e v|
$$

For each $n \in \mathbb{N}$, let

$$
\begin{aligned}
& A_{n}=\left\{t \in T:\left|z_{n}(t)\right| \geq \frac{\varepsilon_{0}}{8} \max _{e}\left|x_{0}(t)+e z_{n}(t)\right|\right\}, \\
& z_{n}^{(1)}: z_{n}^{(1)}(t)=z_{n}(t)\left(t \notin A_{n}\right), \quad z_{n}^{(1)}(t)=0\left(t \in A_{n}\right), \\
& z_{n}^{(2)}: z_{n}^{(2)}(t)=0\left(t \notin A_{n}\right), \quad z_{n}^{(2)}(t)=z_{n}(t)\left(t \in A_{n}\right) .
\end{aligned}
$$

It is easy to see that $z_{n}=z_{n}^{(1)}+z_{n}^{(2)}, \forall n \in \mathbb{N}$. Since $\left|\lambda_{n}\right| \rightarrow 1$ when $n \rightarrow \infty$, the following inequalities

$$
\begin{aligned}
& \left\|z_{n}^{(1)}\right\|_{\Phi, p} \\
& \quad=\inf _{k>0} \frac{1}{k}\left\{1+\left[\int_{t \notin A_{n}} \Phi\left(t, k\left|z_{n}(t)\right|\right) d t\right]^{p}\right\}^{1 / p} \\
& \quad \leq \inf _{k>0} \frac{1}{k}\{1+ \\
& \quad \leq \frac{\varepsilon_{0}}{8}\left\|\max _{e}\left|x_{0}+e z_{n}\right|\right\|_{\Phi, p} \\
& \quad \leq \frac{\varepsilon_{0}}{8}\left\|\sum_{e}\left|x_{0}+e z_{n}\right|\right\|_{\Phi, p} \\
& \quad \leq \frac{\varepsilon_{0}}{2\left|\lambda_{n}\right|}<\frac{3 \varepsilon_{0}}{4}
\end{aligned}
$$

hold for $n$ large enough.

Therefore, $\left\|z_{n}^{(2)}\right\|_{\Phi, p}>\varepsilon_{0} / 4$ which shows that $\mu\left(A_{n}\right)>0$ for $n$ large enough. Furthermore, for any $t \in A_{n}$, we have

$$
\left|x_{0}(t)\right| \leq \frac{1-2 \delta_{0}}{4} \Sigma_{e}\left|x_{0}(t)+e z_{n}(t)\right| \text {. }
$$

To complete the proof, we consider the following two cases.

(I) One has $k_{n} \rightarrow \infty(n \rightarrow \infty)$, where $k_{n} \in K_{p}((1 / 4)$ $\left.\Sigma_{e}\left|x_{0}+e z_{n}\right|\right)$. 
For each $n \in \mathbb{N}$, we get

$$
\begin{aligned}
& 1=\left\|x_{0}\right\|_{\Phi, p} \\
& \leq \frac{1}{k_{n}}\left\{1+I_{\Phi}^{p}\left(k_{n} x_{0}\right)\right\}^{1 / p} \\
& =\frac{1}{k_{n}}\left\{1+\left[\int_{t \in A_{n}} \Phi\left(t, k_{n}\left|x_{0}(t)\right|\right) d t\right.\right. \\
& \left.\left.+\int_{t \notin A_{n}} \Phi\left(t, k_{n}\left|x_{0}(t)\right|\right) d t\right]^{p}\right\}^{1 / p} \\
& \leq \frac{1}{k_{n}}\left\{1+\left[\int _ { t \in A _ { n } } \Phi \left(t, k_{n}\left(\frac{1-2 \delta_{0}}{4}\right.\right.\right.\right. \\
& \left.\left.\times \Sigma_{e}\left|x_{0}(t)+e z_{n}(t)\right|\right)\right) d t \\
& +\int_{t \notin A_{n}} \Phi\left(t, k_{n}\right. \\
& \left.\left.\left.\times\left(\frac{1}{4} \Sigma_{e}\left|x_{0}(t)+e z_{n}(t)\right|\right)\right) d t\right]^{p}\right\}^{1 / p} \\
& \leq \frac{1}{k_{n}}\{1 \\
& +\left[\left(1-2 \delta_{0}\right)\right. \\
& \times \int_{t \in A_{n}} \Phi\left(t, k_{n}\left(\frac{1}{4} \Sigma_{e}\left|x_{0}(t)+e z_{n}(t)\right|\right)\right) d t \\
& +\int_{t \notin A_{n}} \Phi\left(t, k_{n}\right. \\
& \left.\left.\left.\times\left(\frac{1}{4} \Sigma_{e}\left|x_{0}(t)+e z_{n}(t)\right|\right)\right) d t\right]^{p}\right\}^{1 / p} \\
& =\frac{1}{k_{n}}\{1 \\
& +\left[I_{\Phi}\left(k_{n}\left(\frac{1}{4} \Sigma_{e}\left|x_{0}+e z_{n}\right|\right)\right)-2 \delta_{0}\right. \\
& \times \int_{t \in A_{n}} \Phi\left(t, k_{n}\right. \\
& \left.\left.\left.\times\left(\frac{1}{4} \Sigma_{e}\left|x_{0}(t)+e z_{n}(t)\right|\right)\right) d t\right]^{p}\right\}^{1 / p} .
\end{aligned}
$$

Furthermore, we notice that

$$
\begin{aligned}
\int_{t \in A_{n}} \Phi\left(t, k_{n}\left(\frac{1}{4} \Sigma_{e}\left|x_{0}(t)+e z_{n}(t)\right|\right)\right) d t \\
\geq \int_{t \in A_{n}} \Phi\left(t, k_{n}\left|z_{n}(t)\right|\right) d t \\
\geq\left[k_{n}^{p}\left\|z_{n}^{(2)}\right\|_{\Phi, p}^{p}-1\right]^{1 / p} \\
\geq\left[\left(\frac{\varepsilon_{0}}{4} k_{n}\right)^{p}-1\right]^{1 / p} .
\end{aligned}
$$

Since $k_{n} \rightarrow \infty$ when $n \rightarrow \infty$, we can see that the inequality $\left(\left(\varepsilon_{0} / 4\right) k_{n}\right)^{p}-1>0$ holds for $n$ large enough. Moreover, we find that

$$
\begin{aligned}
& I_{\Phi}\left(k_{n}\left(\frac{1}{4} \Sigma_{e}\left|x_{0}+e z_{n}\right|\right)\right) \\
& \quad \geq I_{\Phi}\left(k_{n} x_{0}\right) \\
& \quad \geq\left(k_{n}^{p}-1\right)^{1 / p} \geq 2 \delta_{0}\left(\left(\frac{\varepsilon_{0}}{4} k_{n}\right)^{p}-1\right)^{1 / p} .
\end{aligned}
$$

Then we deduce

$$
\begin{aligned}
& 1=\left\|x_{0}\right\|_{\Phi, p} \\
& \leq \frac{1}{k_{n}}\left\{1+\left[I_{\Phi}\left(k_{n}\left(\frac{1}{4} \Sigma_{e}\left|x_{0}+e z_{n}\right|\right)\right)-2 \delta_{0}\right.\right. \\
& \times \int_{t \in A_{n}} \Phi\left(t, k_{n}\right. \\
& \left.\left.\left.\times\left(\frac{1}{4} \Sigma_{e}\left|x_{0}(t)+e z_{n}(t)\right|\right)\right) d t\right]^{p}\right\}^{1 / p} \\
& \leq \frac{1}{k_{n}}\left\{1+\left[I_{\Phi}\left(k_{n}\left(\frac{1}{4} \Sigma_{e}\left|x_{0}+e z_{n}\right|\right)\right)\right.\right. \\
& \left.\left.-2 \delta_{0}\left(\left(\frac{\varepsilon_{0}}{4} k_{n}\right)^{p}-1\right)^{1 / p}\right]^{p}\right\}^{1 / p} \\
& <\frac{1}{k_{n}}\left\{1+I_{\Phi}^{p}\left(k_{n}\left(\frac{1}{4} \Sigma_{e}\left|x_{0}+e z_{n}\right|\right)\right)\right\}^{1 / p}=1 \text {, }
\end{aligned}
$$

a contradiction.

(II) One has $k_{n} \rightarrow k_{0}(n \rightarrow \infty)$, where $k_{n} \in$ $K_{p}\left((1 / 4) \Sigma_{e}\left|x_{0}+e z_{n}\right|\right), k_{0} \in \mathbb{R}$.

Then for each $n \in \mathbb{N}$,

$$
\begin{aligned}
\frac{1}{\lambda_{n}} & \geq\left\|\frac{1}{4} \Sigma_{e}\left|x_{0}+e z_{n}\right|\right\|_{\Phi, p} \\
& =\frac{1}{k_{n}}\left\{1+I_{\Phi}^{p}\left(\frac{k_{n}}{4} \Sigma_{e}\left|x_{0}+e z_{n}\right|\right)\right\}^{1 / p} \\
& \geq \frac{1}{k_{n}}\left\{1+I_{\Phi}^{p}\left(k_{n} x_{0}\right)\right\}^{1 / p} \geq\left\|x_{0}\right\|_{\Phi, p}=1 .
\end{aligned}
$$

Let $n \rightarrow \infty$, we deduce that $1=\left(1 / k_{0}\right)\left\{1+I_{\Phi}^{p}\left(k_{0} x_{0}\right)\right\}^{1 / p}=$ $\left\|x_{0}\right\|_{\Phi, p}$. 
From (I), we obtain that

$$
\begin{aligned}
& 1=\left\|x_{0}\right\|_{\Phi, p} \\
& \leq \frac{1}{k_{n}}\left\{1+\left[I_{\Phi}\left(k_{n}\left(\frac{1}{4} \Sigma_{e}\left|x_{0}+e z_{n}\right|\right)\right)-2 \delta_{0}\right.\right. \\
& \quad \times \int_{t \in A_{n}} \Phi\left(t, k_{n}\right. \\
&\left.\left.\left.\quad \times\left(\frac{1}{4} \Sigma_{e}\left|x_{0}(t)+e z_{n}(t)\right|\right)\right) d t\right]^{p}\right\}^{1 / p} .
\end{aligned}
$$

Now we consider the following two subcases.

(a) Consider $\int_{t \in A_{n}} \Phi\left(t, k_{n}\left((1 / 4) \Sigma_{e}\left|x_{0}(t)+e z_{n}(t)\right|\right)\right) d t>0$ for some $n \in \mathbb{N}$.

Then we obtain

$$
\begin{aligned}
& 1=\left\|x_{0}\right\|_{\Phi, p} \\
& \leq \frac{1}{k_{n}}\left\{1+\left[I_{\Phi}\left(k_{n}\left(\frac{1}{4} \Sigma_{e}\left|x_{0}+e z_{n}\right|\right)\right)-2 \delta_{0}\right.\right. \\
& \times \int_{t \in A_{n}} \Phi\left(t, k_{n}\right. \\
& \left.\left.\left.\times\left(\frac{1}{4} \Sigma_{e}\left|x_{0}(t)+e z_{n}(t)\right|\right)\right) d t\right]^{p}\right\}^{1 / p} \\
& <\frac{1}{k_{n}}\left\{1+I_{\Phi}^{p}\left(k_{n}\left(\frac{1}{4} \Sigma_{e}\left|x_{0}+e z_{n}\right|\right)\right)\right\}^{1 / p}=1,
\end{aligned}
$$

a contradiction.

(b) Consider $\int_{t \in A_{n}} \Phi\left(t, k_{n}\left((1 / 4) \Sigma_{e}\left|x_{0}(t)+e z_{n}(t)\right|\right)\right) d t=0$ for any $n \in \mathbb{N}$.

For $n$ large enough, we observe that

$$
k_{n}>\frac{1-2 \delta_{0}}{1-\delta_{0}} k_{0} .
$$

Hence, we get

$$
\begin{aligned}
0 & =\int_{t \in A_{n}} \Phi\left(t, k_{n}\left(\frac{1}{4} \Sigma_{e}\left|x_{0}(t)+e z_{n}(t)\right|\right)\right) d t \\
& \geq \int_{t \in A_{n}} \Phi\left(t,\left|\frac{k_{n}}{1-2 \delta_{0}} x_{0}(t)\right|\right) d t \\
& \geq \int_{t \in A_{n}} \Phi\left(t,\left|\frac{k_{0}}{1-\delta_{0}} x_{0}(t)\right|\right) d t \geq 0 .
\end{aligned}
$$

Thus, we see the equality $\int_{t \in A_{n}} \Phi\left(t,\left|\left(k_{0} /\left(1-\delta_{0}\right)\right) x_{0}(t)\right|\right) d t=0$ holds for $n$ large enough. It follows that

$$
\left|\frac{k_{0}}{1-\delta_{0}} x_{0}(t)\right| \leq e(t), \quad \mu \text {-a.e. } t \in A_{n}
$$

since $\mu\left(A_{n}\right)>0$ for $n$ large enough.
On the other hand, by (3), we deduce that

$$
\left|k_{0} x_{0}(t)\right| \geq e(t), \quad \mu \text {-a.e. } t \in A_{n} \text {. }
$$

Hence, we get a contradiction:

$$
\left|\frac{k_{0}}{1-\delta_{0}} x_{0}(t)\right| \geq \frac{e(t)}{1-\delta_{0}}>e(t), \quad \mu \text {-a.e. } t \in A_{n} \text {. }
$$

Theorem 4. Assume $1 \leq p<\infty$; then the following assertions are equivalent:

(1) $L_{\Phi, p}$ is complex midpoint locally uniformly convex;

(2) $L_{\Phi, p}$ is complex strictly convex;

(3) $e(t)=0$ for $\mu$-a.e. $t \in T$.

Proof. The implication (1) $\Rightarrow(2)$ is trivial. Now assume that $L_{\Phi, p}$ is complex strictly convex. If $\mu\{t \in T: e(t)>0\}>0$, let $T_{0}=\{t \in T: e(t)>0\}$ and it is not difficult to find an element $x \in S\left(L_{\Phi, p}\right)$ satisfying supp $x=T \backslash T_{0}$. Take $k \in K_{p}(x)$, and define

$$
y(t)= \begin{cases}\frac{e(t)}{2 k} & \text { for } t \in T_{0} \\ x(t) & \text { for } t \in T \backslash T_{0} .\end{cases}
$$

Obviously, $\|y\|_{\Phi, p} \geq\|x\|_{\Phi, p}=1$. On the other hand,

$$
\begin{aligned}
\|y\|_{\Phi, p} \leq \frac{1}{k}\{1 & +\left[\int_{t \in T_{0}} \Phi\left(t, \frac{e(t)}{2}\right) d t\right. \\
& \left.\left.+\int_{T \backslash T_{0}} \Phi(t, k x(t)) d t\right]^{p}\right\}^{1 / p} \\
=\frac{1}{k}\left(1+I_{\Phi}^{p}(k x)\right)^{1 / p}=1 . &
\end{aligned}
$$

Thus, $\|y\|_{\Phi, p}=(1 / k)\left(1+I_{\Phi}^{p}(k y)\right)^{1 / p}=1$. However, for $t \epsilon$ $T_{0}$, we find $k|y(t)|=e(t) / 2<e(t)$, which implies $y \in S\left(L_{\Phi, p}\right)$ is not a complex extreme point of $B\left(L_{\Phi, p}\right)$ from Theorem 3 .

$(3) \Rightarrow(1)$. Suppose that $x \in S\left(L_{\Phi, p}\right)$ is not a complex strongly extreme point of $B\left(L_{\Phi, p}\right)$. It follows from Theorem 3 that $\mu\left\{t \in T: k_{0}|x(t)|<e(t)\right\}>0$ for some $k_{0} \in K_{p}(x)$, consequently $\mu\{t \in T: e(t)>0\}>0$ which is a contradiction.

Remark 5. If $p=\infty$ then $p$-Amemiya norm equals Luxemburg norm, the problem of complex convexity of MusielakOrlicz function spaces equipped with the Luxemburg norm has been investigated in [8].

\section{Conflict of Interests}

The authors declare that there is no conflict of interests regarding the publication of this paper. 


\section{Acknowledgments}

This work is supported by Grants from Heilongjiang Provincial Natural Science Foundation for Youths (no. QC2013C001), Natural Science Foundation of Heilongjiang Educational Committee (no. 12531099), Youth Science Fund of Harbin University of Science and Technology (no. 2011YF002), and Tianyuan Funds of the National Natural Science Foundation of China (no. 11226127).

\section{References}

[1] O. Blasco and M. Pavlović, "Complex convexity and vectorvalued Littlewood-Paley inequalities," Bulletin of the London Mathematical Society, vol. 35, no. 6, pp. 749-758, 2003.

[2] C. Choi, A. Kamińska, and H. J. Lee, "Complex convexity of Orlicz-Lorentz spaces and its applications," Bulletin of the Polish Academy of Sciences: Mathematics, vol. 52, no. 1, pp. 19-38, 2004.

[3] H. Hudzik and A. Narloch, "Relationships between monotonicity and complex rotundity properties with some consequences," Mathematica Scandinavica, vol. 96, no. 2, pp. 289-306, 2005.

[4] H. J. Lee, "Monotonicity and complex convexity in Banach lattices," Journal of Mathematical Analysis and Applications, vol. 307, no. 1, pp. 86-101, 2005.

[5] H. J. Lee, "Complex convexity and monotonicity in QuasiBanach lattices," Israel Journal of Mathematics, vol. 159, no. 1, pp. 57-91, 2007.

[6] M. M. Czerwińska and A. Kamińska, "Complex rotundities and midpoint local uniform rotundity in symmetric spaces of measurable operators," Studia Mathematica, vol. 201, no. 3, pp. 253-285, 2010.

[7] E. Thorp and R. Whitley, "The strong maximum modulus theorem for analytic functions into a Banach space," Proceedings of the American Mathematical Society, vol. 18, pp. 640-646, 1967.

[8] L. Chen, Y. Cui, and H. Hudzik, "Criteria for complex strongly extreme points of Musielak-Orlicz function spaces," Nonlinear Analysis: Theory, Methods \& Applications, vol. 70, no. 6, pp. 2270-2276, 2009.

[9] S. Chen, Geometry of Orlicz Spaces, Dissertationes Mathematicae, 1996.

[10] Y. Cui, L. Duan, H. Hudzik, and M. Wisła, "Basic theory of $p$ Amemiya norm in Orlicz spaces $(1 \leq p \leq \infty)$ : extreme points and rotundity in Orlicz spaces endowed with these norms," Nonlinear Analysis: Theory, Methods \& Applications, vol. 69, no. 5-6, pp. 1796-1816, 2008. 


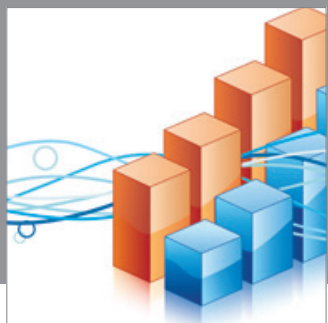

Advances in

Operations Research

mansans

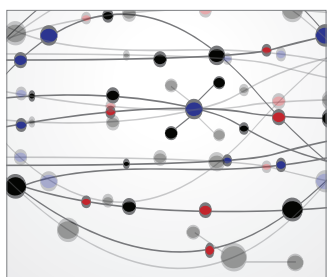

The Scientific World Journal
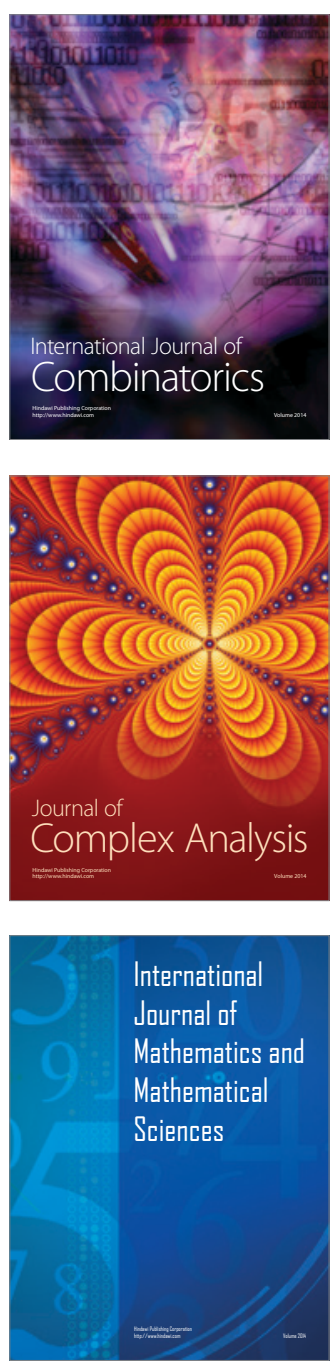
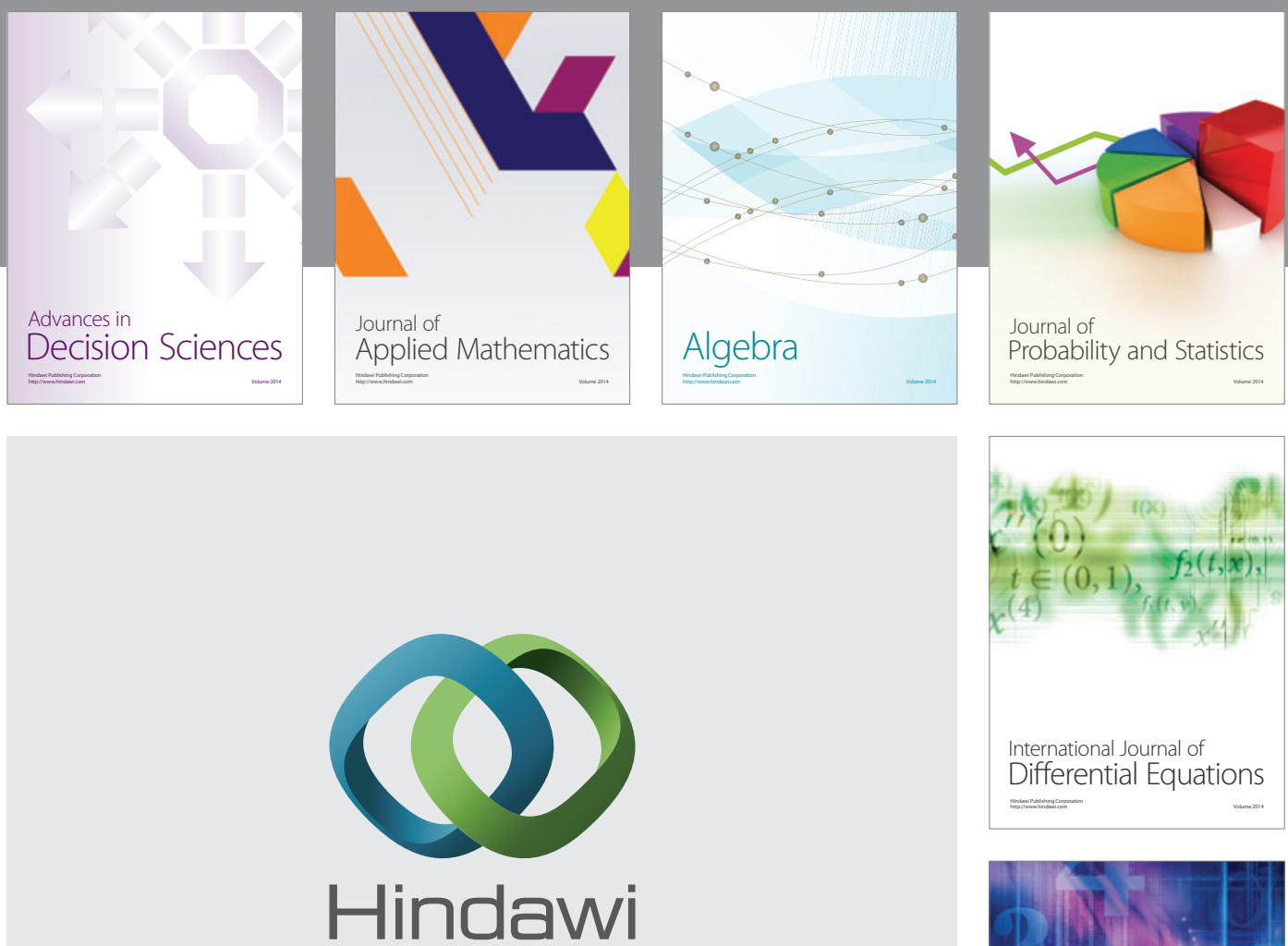

Submit your manuscripts at http://www.hindawi.com
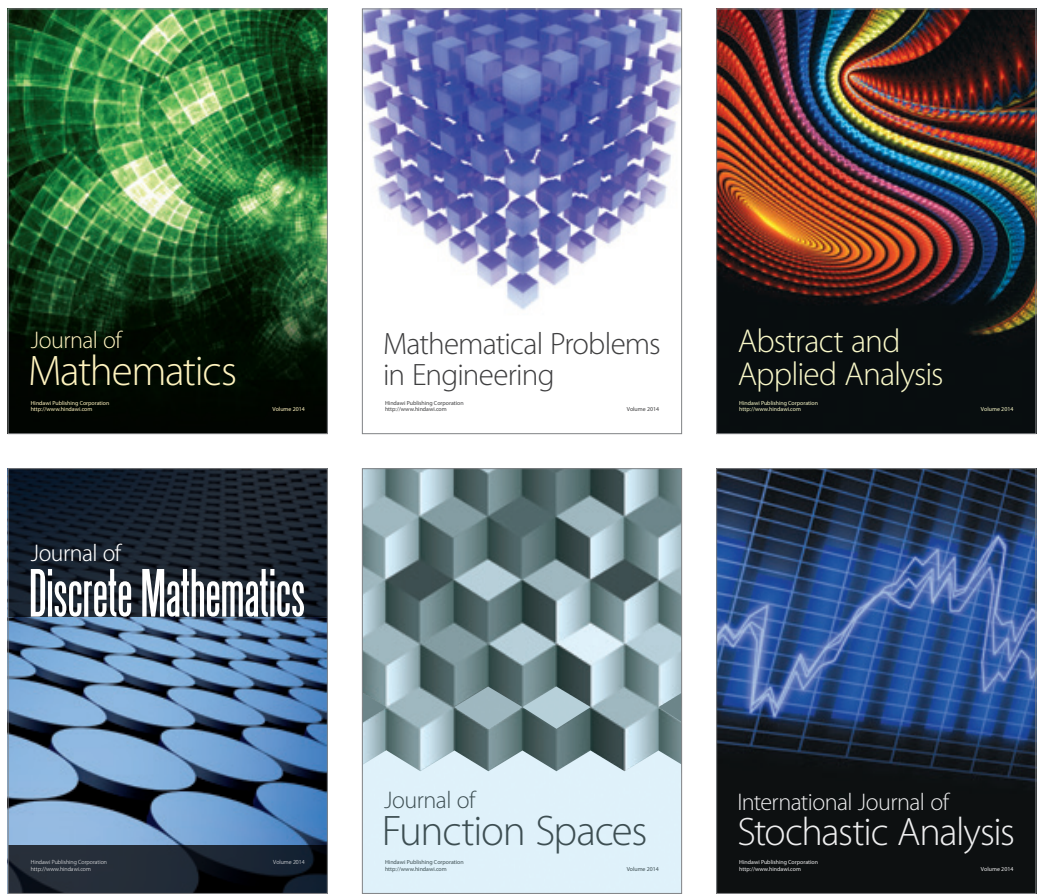

Journal of

Function Spaces

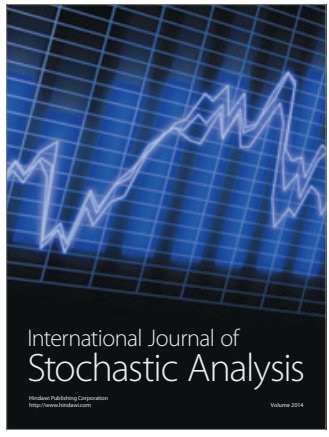

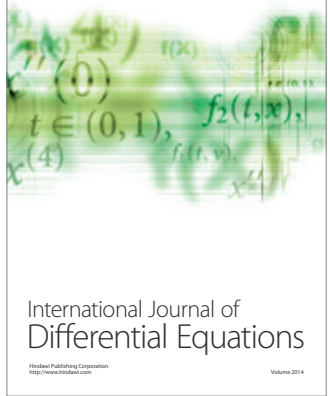
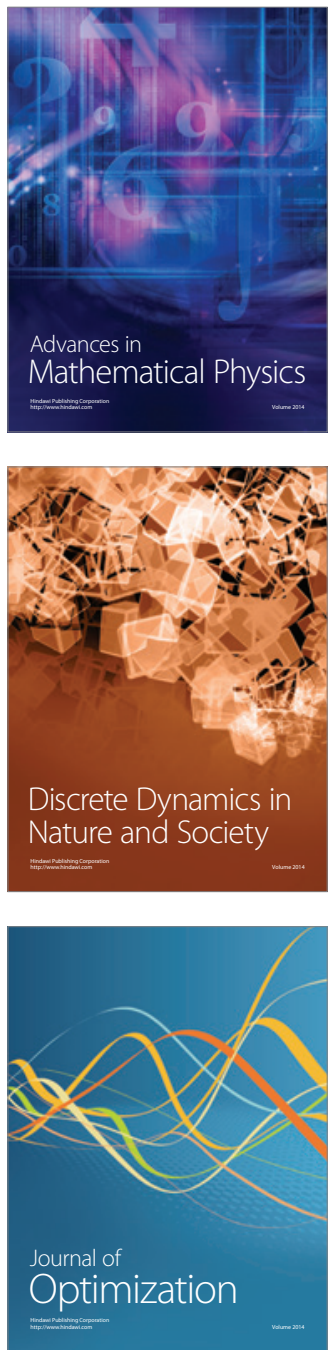